\title{
A simple dual ascent algorithm for the multilevel facility location problem
}

\author{
A.F. Bumb, W.Kern \\ Faculty of Mathematical Sciences, University of Twente, P.O. Box 217, 7500 AE \\ Enschede, The Netherlands, \\ email: a.f.bumb@math.utwente.nl,kern@math.utwente.nl
}

\begin{abstract}
We present a simple dual ascent method for the multilevel facility location problem which finds a solution within 6 times the optimum for the uncapacitated case and within 12 times the optimum for the capacitated one. The algorithm is deterministic and based on the primal-dual techinique.
\end{abstract}

\section{Introduction}

This paper concernes with the multilevel facility location problem. Being an extension of the uncapacitated facility location problem, which is known to be Max SNP-hard (see[2]), this problem is Max SNP-hard as well. The first approximation algorithms for the multilevel facility location problem were developed by Shmoys, Tardos \& Aardal [5] and Aardal, Chudak \& Shmoys [1] and were based on rounding of an LP solution to an integer one. The performance guarantee of these algorithms were 3.16 , respectively 3 . The first combinatorial algorithm for the multilevel facility location problem was developed by Meyerson, Munagala\&Plotkin [4], and finds a solution within $O(\log |D|)$ the optimum, where $D$ is the set of demand points. Using an idea from [3], we present a simple greedy (dual ascent) method for the multilevel facility location problem that finds a solution within 6 times the optimum. The algorithm extends to a capacitated variant of the problem, when each facility can serve only a certain number of demand points, with an increase of the performance guarantee to 12 .

Preprint submitted to Elsevier Science

26 April 2001 


\section{The metric multilevel uncapacitated facility location problem}

Consider a complete $(k+1)$-partite graph $G=(V, E)$ with $V=V_{0} \cup \ldots \cup V_{k}$ and $E=\bigcup_{l=1}^{k} V_{l-1} \times V_{l}$. The set $D=V_{0}$ is the set of demand nodes and $F=V_{1} \cup \ldots \cup V_{k}$ is the set of possible facility locations (at level $1, \ldots, k$ ). We are given edge costs $c \in R_{+}^{E}$ and opening costs $f \in R_{+}^{F}$ ( i.e., opening a facility at $i \in F$ incurs a cost $f_{i} \geq 0$ ). We assume that $c$ is induced by a metric on $V$. Without loss of generality we can assume that there are no edges of cost 0 . Denote by $P$ the set of paths of length $k-1$ joining some node in $V_{1}$ to some node in $V_{k}$. If $j \in D$ and $p=\left(v_{1}, \ldots, v_{k}\right) \in P$, we let $j p$ denote the path $\left(j, v_{1}, \ldots, v_{k}\right)$. As usual $c(p)$ and $c(j p)$ denote the length of $p$ resp. $j p$ (with respect to $c$ ).

The corresponding UFL problem can now be stated as follows: Determine for each $j \in D$ a path $p_{j} \in P$ ( along "open facilities") so as to minimize $\sum_{j \in D} c\left(j p_{j}\right)+f\left(\bigcup_{j \in D} p_{j}\right)$. In this setting we assume that each $j \in D$ has a demand of one unit to be shipped along $p_{j}$. Our results easily extend to arbitrary positive demands.

To derive an integer programming formulation of the UFL problem, we introduce the $0-1$ variables $y_{i}(i \in F)$ to indicate whether $i \in F$ is open and the $0-1$ variables $x_{j p}(j \in D, p \in P)$ to indicate whether $j$ is served along $p$. We let $c(x):=\sum_{p \in P} \sum_{j \in D} c_{j p} x_{j p}$ and $f(y):=\sum_{i \in F} f_{i} y_{i}$.

The UFL problem is now equivalent to

$$
\begin{aligned}
\operatorname{minimize} & c(x)+f(y) \\
\text { subject to } & \sum_{p \in P} x_{j p}=1, \quad \text { for each } j \in D \\
& \sum_{p \ni i} x_{j p} \leq y_{i}, \quad \text { for each } i \in F, j \in D \\
& x_{j p}, y_{i} \in\{0,1\}, \quad \text { for each } p \in P, j \in D, \quad i \in F
\end{aligned}
$$

Denote with $(P)$ the $L P$-relaxation of the above integer program obtained by allowing $(x, y)$ to be positive reals.

\section{The dual ascent algorithm}

Introducing dual variables $v_{j}$ and $t_{i j}$ corresponding to constraints (1) and (2) in $(P)$, the dual of $(P)$ can be written as

$$
\operatorname{maximize} \sum_{j \in D} v_{j}
$$




$$
\begin{array}{cc}
\text { subject to } \quad v_{j}-\sum_{i \in p} t_{i j} \leq c(j p), & \text { for each } p \in P, j \in D \\
& \sum_{j \in D} t_{i j} \leq f_{i}, \quad \text { for each } i \in F \quad \text { for each } i \in F, j \in D \\
& t_{i j} \geq 0, \quad j \in
\end{array}
$$

We first describe how to construct the dual solution $(v, t)$. To this end,we introduce the following notation w.r.t. an arbitrary feasible solution $(v, t)$ of $(D)$ : A facility $i \in F$ is fully paid when $\sum_{j \in D} t_{i j}=f_{i}$. A demand point $j \in D$ reaches $i_{l} \in V_{l}$ if for some path $p=\left(i_{1}, \ldots, i_{l}\right)$ from $V_{1}$ to $i_{l}$ all facilities $i_{1}, \ldots i_{l-1}$ are fully paid and $v_{j}=c_{j p}+\sum_{i \in p} t_{i j}$. If, in addition, also $i_{l}$ is fully paid, we say that $j$ leaves $i_{l}$ or, in case $l=k$, that $j$ gets connected ( along $p$ to $i_{k} \in V_{k}$ ).

Our algorithm for constructing the dual solution is a dual ascent method, generalizing the approach in [3].

We start with $v \equiv t \equiv 0$ and increase all $v_{j}$ uniformly ( with unit speed" ). Until all demand points get connected we proceed as follows. When some $j \in D$ reaches a not fully paid node $i \in F$, we start increasing $t_{i j}$ with unit speed, until $f_{i}$ is fully paid and $j$ leaves $i$. We stop increasing $v_{j}$ when $j$ gets connected.The algorithm maintains the invariant that at time $T$ the dual variables $v_{j}$ that are still being raised are all equal to $T$.

Let $(v, t)$ denote the final dual solution. For each fully paid facility $i \in V_{l}$, $l \geq 2$,denote by $T_{i}$ the time when facility $i$ became fully paid. The predecessor of $i$ will be the facility in the level $l-1$ via which $i$ was for the first time reached by a demand point, i.e.,

$\operatorname{Pred}(i)=\left\{i^{\prime} \in V_{l-1} \mid i^{\prime}\right.$ is fully paid and $\left.T_{i^{\prime}}+c_{i^{\prime} i}=\min _{i^{\prime \prime} \in V_{l-1} i^{\prime \prime} \text { fully paid }}\left(T_{i^{\prime \prime}}+c_{i^{\prime \prime} i}\right)\right\}$

(Ties are broken arbitrarily.) The predecessor of a fully paid facility $i \in V_{1}$ will be its closest demand point. We can define the time $T_{\text {Pred }(i)}=0$.

For all fully paid facilities $i$ in the $k-t h$ level denote by $j_{i} p_{i}=\left(i_{1}, \ldots i_{k}\right)$ the path through the following points:

- $i_{k}=i$

- $i_{l}=\operatorname{Pred}\left(i_{l+1}\right)$, for each $1 \leq l \leq k-1$

- $j_{i}=\operatorname{Pred}\left(i_{1}\right)$.

We will call the neighborhood of $i$ the set of demand nodes contributing to $p_{i}$ i.e., $N_{i}=\left\{j \in D \mid t_{i^{\prime} j}>0\right.$ for some $\left.i^{\prime} \in p_{i}\right\}$. Since each $j \in D$ gets connected we may fix for each $j \in D$ a connecting path $\widetilde{p_{j}} \in P$ of fully paid facilities ( ties are broken arbitrarily). We now describe how to construct a corresponding primal solution $(x, y)$.

Suppose there are $r$ fully paid facilities in the last level. Order them according to nondecreasing $T$-values, say $T_{1} \leq \ldots T_{r}$. Construct greedily a set $C \subseteq V_{k}$ of centers which have parewise disjoint neighborhoods and assign each $j \in D$ 
to some center $i_{0} \in C$ in the following way: For each fully paid facility $i$ in the last level check if there is a center $i_{0} \leq i$ such that $N_{i} \cap N_{i_{0}} \neq \varnothing$. If such an $i_{0}$ exist, assign to $p_{i_{0}}$ all demand nodes $j \in D$ with $i \in \widetilde{p_{j}}$. Otherwise add $i$ to $C$ and assign to $p_{i}$ all the demand nodes $j \in D$ with $i \in \widetilde{p_{j}}$.

The primal solution $(x, y)$ is obtained as follows:

$$
x_{j p}:=\left\{\begin{array}{l}
1 \text { if } p=p_{i} \text { and } j \text { was assigned to } i \in V_{k} \\
0 \text { otherwise }
\end{array}\right.
$$

and

$$
y_{i}:=\left\{\begin{array}{l}
1 \text { if } i \text { is on a central path } \\
0 \text { otherwise }
\end{array}\right.
$$

Theorem 1 The above primal solution $(x, y)$ satisfies

$$
c(x)+f(y) \leq 6 \sum_{j \in D} \nu_{j}
$$

implying that the performance guarantee of algorithm is 6 .

NOTE: The greedy dual ascent algorithm combined with a technique based on Lagrangian multipliers yields a 12 approximation algorithm for the capacitated variant of the multilevel facility location problem in which each facility can serve only a certain number of demand points.

\section{References}

[1] K.I.Aardal, F.Chudak and D.B.Shmoys. A 3-approximation algorithm for the k-level uncapacitated facility location problem. Information Processing Letters, 72, pages $161-167,1999$

[2] S.Guha and S. Khuller. Greedy strikes back: improved facility algorithms. Proceedings of the 9th Annual ACM-SIAM Symposium on Discrete Algorithms, pages $649-657,1998$

[3] K.Jain and V.V.Vazirani. Primal-dual approximation algorithms for metric facility location and $k$-median problems. Proceedings of the Twenty-Ninth Annual IEEE Symposium on Foundations of Computer Science, 1999

[4] A. Meyerson, K. Munagala, S. Plotkin. Cost distance: Two metric network design. Proceedings of the 41-th IEEE Symposium on Foundation of Computer Science, 2000

[5] D. B. Shmoys, E. Tardos and K. I. Aardal. Approximation algorithms for facility location problems. Proceedings of the 29th Annual ACM Symposium on Theory of Computing, pages 265-274, 1997 